\title{
On an axiomatization of the quasi-arithmetic mean values without the symmetry axiom
}

\author{
Jean-Luc Marichal \\ Department of Management, FEGSS, University of Liège \\ Boulevard du Rectorat 7 - B31, B-4000 Liège, Belgium \\ jl.marichal[at]ulg.ac.be
}

Revised version, November 23, 1998

\begin{abstract}
Kolmogoroff and Nagumo proved that the quasi-arithmetic means correspond exactly to the decomposable sequences of continuous, symmetric, strictly increasing in each variable and reflexive functions. We replace decomposability and symmetry in this characterization by a generalization of the decomposability.
\end{abstract}

AMS (1991) subject classification: Primary 39B12, 39B22, Secondary $26 B 35$.

\section{Introduction}

A considerable amount of literature about the concept of mean (or average) and the properties of several means (such as the median, the arithmetic mean, the geometric mean, the power mean, the harmonic mean, etc.) has been already produced in the 19th century and has often treated the significance and the interpretation of these specific aggregation functions.

Cauchy [5] considered in 1821 the mean of $n$ independent variables $x_{1}, \ldots, x_{n}$ as a function $M\left(x_{1}, \ldots, x_{n}\right)$ which should be internal to the set of $x_{i}$ values:

$$
\min \left\{x_{1}, \ldots, x_{n}\right\} \leq M\left(x_{1}, \ldots, x_{n}\right) \leq \max \left\{x_{1}, \ldots, x_{n}\right\} .
$$

The concept of mean as an average is usually ascribed to Chisini [6], who gives in 1929 the following definition (p. 108):

Let $y=g\left(x_{1}, \ldots, x_{n}\right)$ be a function of $n$ independent variables $x_{1}, \ldots, x_{n}$ representing homogeneous quantities. A mean of $x_{1}, \ldots, x_{n}$ with respect to the function $g$ is a number $M$ such that, if each of $x_{1}, \ldots, x_{n}$ is replaced by $M$, the function value is unchanged, that is,

$$
g(M, \ldots, M)=g\left(x_{1}, \ldots, x_{n}\right) .
$$


When $g$ is considered as the sum, the product, the sum of squares, the sum of inverses, the sum of exponentials, or is proportional to $\left[\left(\sum_{i} x_{i}^{2}\right) /\left(\sum_{i} x_{i}\right)\right]^{1 / 2}$ as for the duration of oscillations of a composed pendulum of $n$ elements of same weights, the solution of Chisini's equation corresponds respectively to the arithmetic mean, the geometric mean, the quadratic mean, the harmonic mean, the exponential mean and the antiharmonic mean, which is defined as

$$
M\left(x_{1}, \ldots, x_{n}\right)=\left(\sum_{i} x_{i}^{2}\right) /\left(\sum_{i} x_{i}\right) .
$$

Unfortunately, as noted by de Finetti [7, p. 378] in 1931, Chisini's definition is so general that it does not even imply that the "mean" (provided there exists a real and unique solution to the above equation) fulfils the Cauchy's internality property. The following quote from Ricci [13, p. 39] could be considered as another possible criticism to Chisini's view:

... when all values become equal, the mean equals any of them too. The inverse proposition is not true. If a function of several variables takes their common value when all variables coincide, this is not sufficient evidence for calling it a mean. For example, the function

$$
g\left(x_{1}, x_{2}, \ldots, x_{n}\right)=x_{n}+\left(x_{n}-x_{1}\right)+\left(x_{n}-x_{2}\right)+\cdots+\left(x_{n}-x_{n-1}\right)
$$

equals $x_{n}$ when $x_{1}=\cdots=x_{n}$, but it is even greater than $x_{n}$ as long as $x_{n}$ is greater than every other variable.

In 1930, Kolmogoroff [9] and Nagumo [12] considered that the mean should be more than just a Cauchy mean or an average in the sense of Chisini. They defined a mean value to be an infinite sequence of continuous, symmetric and strictly increasing (in each variable) real functions

$$
M_{1}\left(x_{1}\right)=x_{1}, M_{2}\left(x_{1}, x_{2}\right), \ldots, M_{n}\left(x_{1}, \ldots, x_{n}\right), \ldots
$$

satisfying the reflexive law: $M_{n}(x, \ldots, x)=x$ for all $n$ and all $x$, and a certain kind of associative law:

$$
M_{k}\left(x_{1}, \ldots, x_{k}\right)=x \Rightarrow M_{n}\left(x_{1}, \ldots, x_{k}, x_{k+1}, \ldots, x_{n}\right)=M_{n}\left(x, \ldots, x, x_{k+1}, \ldots, x_{n}\right)
$$

for every natural integer $k \leq n$. They proved, independently of each other, that these conditions are necessary and sufficient for the quasi-arithmeticity of the mean, that is, for the existence of a continuous strictly monotonic function $f$ such that $M_{n}$ may be written in the form

$$
M_{n}\left(x_{1}, \ldots, x_{n}\right)=f^{-1}\left[\frac{1}{n} \sum_{i=1}^{n} f\left(x_{i}\right)\right]
$$

for all $n \in \mathbb{N}_{0}$ ( $\mathbb{N}_{0}$ denotes the set of strictly positive integers).

The quasi-arithmetic means (3) comprise most of the algebraic means of common use, and allow one to specify $f$ in relation to operational conditioning, see Table 1 . Some means however do not belong to this family: de Finetti [7, p. 380] observed that the antiharmonic mean is not increasing in each variable and that the median is not associative in the sense of $(2)$.

The above properties defining a mean value seem to be natural enough ${ }^{1}$. For instance, one can readily see that, for increasing means, the reflexivity property is equivalent to Cauchy's internality (1), and both are accepted by all statisticians as requisites for means.

\footnotetext{
${ }^{1}$ Note that Fodor and Marichal [8] generalized the Kolmogoroff-Nagumo's theorem above by relaxing the condition that the means be strictly increasing, requiring only that they be increasing. The family obtained, which has a rather intricate structure, naturally includes the "min" and "max" operations.
} 


\begin{tabular}{|ccc|}
\hline$f(x)$ & $M_{n}\left(x_{1}, \ldots, x_{n}\right)$ & name \\
\hline$x$ & $\frac{1}{n} \sum x_{i}$ & arithmetic \\
$x^{2}$ & $\sqrt{\frac{1}{n} \sum x_{i}^{2}}$ & quadratic \\
$\log x$ & $\sqrt[n]{\prod x_{i}}$ & geometric \\
$x^{-1}$ & $\frac{1}{\frac{1}{n} \sum \frac{1}{x_{i}}}$ & harmonic \\
$x^{\alpha}\left(\alpha \in \mathbb{R}_{0}\right)$ & $\left(\frac{1}{n} \sum x_{i}^{\alpha}\right)^{\frac{1}{\alpha}}$ & power \\
$e^{\alpha x}\left(\alpha \in \mathbb{R}_{0}\right)$ & $\frac{1}{\alpha} \ln \left[\frac{1}{n} \sum e^{\alpha x_{i}}\right]$ & exponential \\
\hline
\end{tabular}

Table 1: Examples of quasi-arithmetic means

Associativity of means (2) has been introduced first in 1926 by Bemporad [4, p. 87] in a characterization of the arithmetic mean. Under reflexivity, this condition seems more natural, for it becomes equivalent to

$$
\begin{aligned}
M_{k}\left(x_{1}, \ldots, x_{k}\right) & =M_{k}\left(x_{1}^{\prime}, \ldots, x_{k}^{\prime}\right) \\
& \Downarrow \\
M_{n}\left(x_{1}, \ldots, x_{k}, x_{k+1}, \ldots, x_{n}\right) & =M_{n}\left(x_{1}^{\prime}, \ldots, x_{k}^{\prime}, x_{k+1}, \ldots, x_{n}\right)
\end{aligned}
$$

which says that the mean does not change when altering some values without modifying their partial mean. More recently, Marichal and Roubens [11] proposed to call this property "decomposability" in order not to confuse it with the classical associativity property.

Observe however that this concept has been defined for symmetric means. When symmetry is not assumed, it is necessary to rewrite the decomposability property in such a way that the first variables are not privileged. Marichal et al. [10] proposed the following general form, called "strong decomposability":

$$
M_{k}\left(x_{i_{1}}, \ldots, x_{i_{k}}\right)=x \quad \Rightarrow \quad M_{n}\left(\sum_{i \in K} x_{i} e_{i}+\sum_{i \notin K} x_{i} e_{i}\right)=M_{n}\left(\sum_{i \in K} x e_{i}+\sum_{i \notin K} x_{i} e_{i}\right)
$$

for every subset $K=\left\{i_{1}, \ldots, i_{k}\right\} \subseteq\{1, \ldots, n\}$ with $i_{1}<\cdots<i_{k}$ ( $e_{i}$ represents the vector of $\{0,1\}^{n}$ in which only the $i$-th component is 1$)$. Of course, under symmetry, decomposability and strong decomposability are equivalent. 
The aim of this paper is to show that symmetry is not necessary in the KolmogoroffNagumo's characterization, provided that decomposability is replaced by strong decomposability $^{2}$. Thus we show that any strongly decomposable sequence $\left(M_{n}\right)_{n \in \mathbb{N}_{0}}$ of continuous, strictly increasing and reflexive functions is a quasi-arithmetic mean value (3).

\section{The result}

We first show that, for any strongly decomposable sequence $\left(M_{n}\right)_{n \in \mathbb{N}_{0}}$ of reflexive functions, the two-place function $M_{2}$ fulfils the bisymmetry functional equation ${ }^{3}$

$$
M_{2}\left(M_{2}\left(x_{1}, x_{2}\right), M_{2}\left(x_{3}, x_{4}\right)\right)=M_{2}\left(M_{2}\left(x_{1}, x_{3}\right), M_{2}\left(x_{2}, x_{4}\right)\right) .
$$

To do this, we need some intermediate results. Let us first introduce the following practical notation: for all $k \in \mathbb{N}_{0}$, we define $k \odot x:=x, \ldots, x$ ( $k$ times). For instance, we have

$$
M_{5}(2 \odot x, 3 \odot y)=M_{5}(x, x, y, y, y) .
$$

Now, we present a technical lemma which is adapted from Nagumo [12, $\S 1]$. It will be very useful as we continue.

Lemma 2.1 Consider a strongly decomposable sequence $\left(M_{n}\right)_{n \in \mathbb{N}_{0}}$ of reflexive functions. Then we have, for all $k, n \in \mathbb{N}_{0}$ with $n \geq 2$,

$$
\begin{aligned}
& M_{k . n}\left(k \odot x_{1}, \ldots, k \odot x_{n}\right)=M_{n}\left(x_{1}, \ldots, x_{n}\right), \\
& M_{k . n}\left(x_{11}, \ldots, x_{1 k} ; \ldots ; x_{n 1}, \ldots, x_{n k}\right)=M_{n}\left(M_{k}\left(x_{11}, \ldots, x_{1 k}\right) ; \ldots ; M_{k}\left(x_{n 1}, \ldots, x_{n k}\right)\right), \\
& M_{n}\left(x_{1}, \ldots, x_{n}\right)=M_{n}\left(x_{n}^{\prime}, \ldots, x_{1}^{\prime}\right), \text { where } x_{j}^{\prime}=M_{n-1}\left(x_{1}, \ldots, x_{j-1}, x_{j+1}, \ldots, x_{n}\right) .
\end{aligned}
$$

Proof. Let us fix $k, n \in \mathbb{N}_{0}$ with $n \geq 2$. We then have

$$
\begin{aligned}
M_{k . n}\left(k \odot x_{1}, \ldots, k \odot x_{n}\right) & =M_{k . n}\left((k . n) \odot M_{n}\left(x_{1}, \ldots, x_{n}\right)\right) \quad(\text { strong decomposability }) \\
& =M_{n}\left(x_{1}, \ldots, x_{n}\right) \quad(\text { reflexivity })
\end{aligned}
$$

which proves (5). Next, we have

$$
\begin{aligned}
& M_{k . n}\left(x_{11}, \ldots, x_{1 k} ; \ldots ; x_{n 1}, \ldots, x_{n k}\right) \\
= & M_{k . n}\left(k \odot M_{k}\left(x_{11}, \ldots, x_{1 k}\right) ; \ldots ; k \odot M_{k}\left(x_{n 1}, \ldots, x_{n k}\right)\right) \quad \text { (strong decomposability) } \\
= & M_{n}\left(M_{k}\left(x_{11}, \ldots, x_{1 k}\right), \ldots, M_{k}\left(x_{n 1}, \ldots, x_{n k}\right)\right) \quad(\text { by }(5))
\end{aligned}
$$

which proves (6). Finally, by (5), we have

$$
M_{n}\left(x_{1}, \ldots, x_{n}\right)=M_{n(n-1)}\left((n-1) \odot x_{1}, \ldots,(n-1) \odot x_{n}\right)
$$

\footnotetext{
${ }^{2}$ One could think that strong decomposability implies symmetry. Nevertheless, the sequence of nonsymmetric means defined for all $n \in \mathbb{N}_{0}$ by $M_{n}\left(x_{1}, \ldots, x_{n}\right)=x_{1}$ is strongly decomposable.

${ }^{3}$ The bisymmetry property (also called mediality) is very easy to handle and has been investigated from the algebraic point of view by using it mostly in structures without the property of associativity - in a certain respect, it has been used as a substitute for associativity and also for symmetry. For a list of references see $[2, \S 6.4]$ (see also [3, Chap. 17]).
} 
and by using strong decomposability with subset $K_{j}=\{j, n+j, 2 n+j, \ldots,(n-2) n+j\}$ for $j=1, \ldots, n$, we obtain

$$
M_{n(n-1)}\left((n-1) \odot x_{1}, \ldots,(n-1) \odot x_{n}\right)=M_{n(n-1)}\left(x_{n}^{\prime}, \ldots, x_{1}^{\prime} ; \ldots ; x_{n}^{\prime}, \ldots, x_{1}^{\prime}\right) .
$$

Therefore, we have

$$
\begin{aligned}
M_{n}\left(x_{1}, \ldots, x_{n}\right) & =M_{n(n-1)}\left(x_{n}^{\prime}, \ldots, x_{1}^{\prime} ; \ldots ; x_{n}^{\prime}, \ldots, x_{1}^{\prime}\right) \\
& =M_{n-1}\left((n-1) \odot M_{n}\left(x_{n}^{\prime}, \ldots, x_{1}^{\prime}\right)\right) \quad(\text { by }(6)) \\
& =M_{n}\left(x_{n}^{\prime}, \ldots, x_{1}^{\prime}\right) \quad(\text { reflexivity })
\end{aligned}
$$

which proves (7).

We now have at hand all the necessary tools to establish that $M_{2}$ is a solution of the bisymmetry equation.

Proposition 2.1 For any strongly decomposable sequence $\left(M_{n}\right)_{n \in \mathbb{N}_{0}}$ of reflexive functions, the two-place function $M_{2}$ fulfils the bisymmetry functional equation (4).

Proof. We have successively,

$$
\begin{aligned}
& M_{2}\left(M_{2}\left(x_{1}, x_{2}\right), M_{2}\left(x_{3}, x_{4}\right)\right) \\
= & M_{4}\left(x_{1}, x_{2}, x_{3}, x_{4}\right) \quad(\text { by }(6)) \\
= & M_{4}\left(M_{2}\left(x_{1}, x_{3}\right), M_{2}\left(x_{2}, x_{4}\right), M_{2}\left(x_{1}, x_{3}\right), M_{2}\left(x_{2}, x_{4}\right)\right) \quad \text { (strong decomposability) } \\
= & M_{2}\left(M_{2}\left(M_{2}\left(x_{1}, x_{3}\right), M_{2}\left(x_{2}, x_{4}\right)\right), M_{2}\left(M_{2}\left(x_{1}, x_{3}\right), M_{2}\left(x_{2}, x_{4}\right)\right) \quad\right. \text { (by (6)) } \\
= & M_{2}\left(M_{2}\left(x_{1}, x_{3}\right), M_{2}\left(x_{2}, x_{4}\right)\right) \quad \text { (reflexivity) }
\end{aligned}
$$

which proves the result.

Now, consider a strongly decomposable sequence $\left(M_{n}\right)_{n \in \mathbb{N}_{0}}$ of continuous, strictly increasing and reflexive functions. Since $M_{2}$ fulfils the bisymmetry equation, it must have a particular form. Actually, it has been proved by Aczél [1] (see also [2, §6.4] and [3, Chap. 17]) that the general continuous, strictly increasing, reflexive real solution of the bisymmetry equation (4) is given by the quasi-linear mean. The statement of this result is formulated as follows.

Theorem 2.1 Let I be any real interval, finite or infinite. A two-place function $M$ : $I^{2} \rightarrow \mathbb{R}$ is continuous, strictly increasing in each variable, reflexive and fulfils the bisymmetric equation (4) if and only if there exists a continuous strictly monotonic function $f: I \rightarrow \mathbb{R}$ and a real number $\theta \in] 0,1[$ such that

$$
M\left(x_{1}, x_{2}\right)=f^{-1}\left[\theta f\left(x_{1}\right)+(1-\theta) f\left(x_{2}\right)\right], \quad \forall x_{1}, x_{2} \in I .
$$

According to this result, $M_{2}$ is of the form (8). By using strong decomposability, we will show later that the number $\theta$ occuring in this form must be $1 / 2$, so that $M_{2}$ is symmetric. Next, we will show that every function $M_{n}$ is also symmetric. Before going on, consider two lemmas. 
Lemma 2.2 If A corresponds to the matrix

$$
\left.A=\left(\begin{array}{ccc}
\theta & \theta & 0 \\
1-\theta & 0 & \theta \\
0 & 1-\theta & 1-\theta
\end{array}\right), \quad \theta \in\right] 0,1[,
$$

then

$$
\lim _{i \rightarrow+\infty} A^{i}=\frac{1}{D}\left(\begin{array}{ccc}
\theta^{2} & \theta^{2} & \theta^{2} \\
\theta(1-\theta) & \theta(1-\theta) & \theta(1-\theta) \\
(1-\theta)^{2} & (1-\theta)^{2} & (1-\theta)^{2}
\end{array}\right)
$$

with $D=\theta^{2}+\theta(1-\theta)+(1-\theta)^{2}$.

Proof. The eigenvalues of $A$ correspond to the solutions of $\operatorname{det}(A-z I)=0$ or

$$
(z-1)\left[\theta(1-\theta)-z^{2}\right]=0 .
$$

Three distinct eigenvalues are obtained: $z_{1}=1, z_{2}=\sqrt{\theta(1-\theta)}, z_{3}=-\sqrt{\theta(1-\theta)}$ and $A$ can be diagonalized:

$$
\Delta=S^{-1} A S=\operatorname{diag}(1, \sqrt{\theta(1-\theta)},-\sqrt{\theta(1-\theta)}) .
$$

We also have the following eigenvectors:

$$
\begin{gathered}
S_{1}=\left(\begin{array}{l}
s_{11} \\
s_{21} \\
s_{31}
\end{array}\right)=\left(\begin{array}{c}
\theta^{2} \\
\theta(1-\theta) \\
(1-\theta)^{2}
\end{array}\right), \quad S_{2}=\left(\begin{array}{c}
s_{12} \\
s_{22} \\
s_{32}
\end{array}\right)=\left(\begin{array}{c}
-\sqrt{\theta} \\
\sqrt{\theta}-\sqrt{1-\theta} \\
\sqrt{1-\theta}
\end{array}\right), \\
S_{3}=\left(\begin{array}{c}
s_{13} \\
s_{23} \\
s_{33}
\end{array}\right)=\left(\begin{array}{c}
\sqrt{\theta} \\
-\sqrt{\theta}-\sqrt{1-\theta} \\
\sqrt{1-\theta}
\end{array}\right) .
\end{gathered}
$$

$A$ can be expressed under the form: $A=S \Delta S^{-1}$ and

$$
A^{i}=S \Delta^{i} S^{-1}, \quad \forall i \in \mathbb{N}_{0} .
$$

Finally, setting $s_{i j}^{\prime}:=\left(S^{-1}\right)_{i j}$, we have

$$
\lim _{i \rightarrow+\infty} A^{i}=S\left(\lim _{i \rightarrow+\infty} \Delta^{i}\right) S^{-1}=\left(\begin{array}{lll}
s_{11}^{\prime} S_{1} & s_{12}^{\prime} S_{1} & s_{13}^{\prime} S_{1}
\end{array}\right) .
$$

Since $S^{-1} S=i d$, we only have to determine $s_{11}^{\prime}, s_{12}^{\prime}, s_{13}^{\prime}$ such that

$$
\left(\begin{array}{lll}
s_{11}^{\prime} & s_{12}^{\prime} & s_{13}^{\prime}
\end{array}\right) S=\left(\begin{array}{lll}
1 & 0 & 0
\end{array}\right)
$$

and we can see that

$$
s_{11}^{\prime}=s_{12}^{\prime}=s_{13}^{\prime}=\frac{1}{D} \text {. }
$$

Lemma 2.3 Consider a strongly decomposable sequence $\left(M_{n}\right)_{n \in \mathbb{N}_{0}}$ of functions. If $M_{2}$ is symmetric then, for all $n \in \mathbb{N}, n>2, M_{n}$ is also symmetric. 
Proof. Let us proceed by induction over $n \geq 2$. Assume that $M_{n}$ is symmetric for a fixed $n \geq 2$. By strong decomposability, we have

$$
\begin{aligned}
M_{n+1}\left(x_{1}, \ldots, x_{n+1}\right) & =M_{n+1}\left(x_{1}, n \odot M_{n}\left(x_{2}, \ldots, x_{n+1}\right)\right) \\
& =M_{n+1}\left(n \odot M_{n}\left(x_{1}, \ldots, x_{n}\right), x_{n+1}\right),
\end{aligned}
$$

and $M_{n+1}$ is also symmetric. Hence the result.

Now, we can turn to the main result. Before stating it, recall the Kolmogoroff-Nagumo's theorem.

Theorem 2.2 Let I be any (finite or infinite) real interval, and $\left(M_{n}\right)_{n \in \mathbb{N}_{0}}$ be a decomposable sequence of continuous, symmetric, strictly increasing and reflexive functions $M_{n}: I^{n} \rightarrow \mathbb{R}$. Then and only then there exists a continuous strictly monotonic function $f: I \rightarrow \mathbb{R}$ such that, for all $n \in \mathbb{N}_{0}$,

$$
M_{n}\left(x_{1}, \ldots, x_{n}\right)=f^{-1}\left[\frac{1}{n} \sum_{i=1}^{n} f\left(x_{i}\right)\right], \quad \forall\left(x_{1}, \ldots, x_{n}\right) \in I^{n} .
$$

As already announced, we show that the symmetry property is not necessary in Theorem 2.2 if we replace decomposability by strong decomposability. The statement is the following.

Theorem 2.3 Let I be any (finite or infinite) real interval, and $\left(M_{n}\right)_{n \in \mathbb{N}_{0}}$ be a strongly decomposable sequence of continuous, strictly increasing and reflexive functions $M_{n}: I^{n} \rightarrow$ $\mathbb{R}$. Then and only then there exists a continuous strictly monotonic function $f: I \rightarrow \mathbb{R}$ such that, for all $n \in \mathbb{N}_{0}$,

$$
M_{n}\left(x_{1}, \ldots, x_{n}\right)=f^{-1}\left[\frac{1}{n} \sum_{i=1}^{n} f\left(x_{i}\right)\right], \quad \forall\left(x_{1}, \ldots, x_{n}\right) \in I^{n} .
$$

Proof. (Sufficiency) Trivial (see Theorem 2.2).

(Necessity) Consider a strongly decomposable sequence $\left(M_{n}\right)_{n \in \mathbb{N}_{0}}$ of continuous, strictly increasing and reflexive functions $M_{n}: I^{n} \rightarrow \mathbb{R}$. By Proposition 2.1, $M_{2}$ fulfils the bisymmetry functional equation (4). By Theorem 2.1, there exists a continuous strictly monotonic function $f: I \rightarrow \mathbb{R}$ and a real number $\theta \in] 0,1[$ such that

$$
M_{2}\left(x_{1}, x_{2}\right)=f^{-1}\left[\theta f\left(x_{1}\right)+(1-\theta) f\left(x_{2}\right)\right], \quad \forall x_{1}, x_{2} \in I .
$$

Define $\Omega:=f(I)=\{f(x) \mid x \in I\}$. The sequence $\left(F_{n}\right)_{n \in \mathbb{N}_{0}}$ defined by

$$
F_{n}\left(z_{1}, \ldots, z_{n}\right):=f\left[M_{n}\left(f^{-1}\left(z_{1}\right), \ldots, f^{-1}\left(z_{n}\right)\right)\right], \quad \forall\left(z_{1}, \ldots, z_{n}\right) \in \Omega^{n}, \forall n \in \mathbb{N}_{0},
$$

is also strongly decomposable and such that each $F_{n}$ is continuous, strictly increasing and reflexive. Moreover, we have

$$
F_{2}\left(z_{1}, z_{2}\right)=\theta z_{1}+(1-\theta) z_{2}, \quad \forall z_{1}, z_{2} \in \Omega
$$

Now, let us show that

$$
F_{3}\left(z_{1}, z_{2}, z_{3}\right)=\frac{1}{D}\left[\theta^{2} z_{1}+\theta(1-\theta) z_{2}+(1-\theta)^{2} z_{3}\right], \quad \forall z_{1}, z_{2}, z_{3} \in \Omega,
$$


with $D=\theta^{2}+\theta(1-\theta)+(1-\theta)^{2}$. We have successively

$$
\begin{aligned}
F_{3}\left(z_{1}, z_{2}, z_{3}\right) & =F_{3}\left(F_{2}\left(z_{1}, z_{2}\right), F_{2}\left(z_{1}, z_{3}\right), F_{2}\left(z_{2}, z_{3}\right)\right) \quad(\text { by }(7)) \\
& =F_{3}\left(\theta z_{1}+(1-\theta) z_{2}, \theta z_{1}+(1-\theta) z_{3}, \theta z_{2}+(1-\theta) z_{3}\right) \quad(\text { by }(9)) \\
& =F_{3}\left(\left(z_{1}, z_{2}, z_{3}\right) A\right)
\end{aligned}
$$

where $A$ is the matrix defined in Lemma 2.2. By iteration, we obtain

$$
\begin{aligned}
F_{3}\left(z_{1}, z_{2}, z_{3}\right) & =F_{3}\left(\left(z_{1}, z_{2}, z_{3}\right) A\right)=F_{3}\left(\left(z_{1}, z_{2}, z_{3}\right) A^{2}\right) \\
& =F_{3}\left(\left(z_{1}, z_{2}, z_{3}\right) A^{i}\right) \quad \forall i \in \mathbb{N}_{0} .
\end{aligned}
$$

We then have

$$
\begin{aligned}
F_{3}\left(z_{1}, z_{2}, z_{3}\right) & =\lim _{i \rightarrow+\infty} F_{3}\left(\left(z_{1}, z_{2}, z_{3}\right) A^{i}\right) \quad(\text { constant numerical sequence) } \\
& =F_{3}\left(\left(z_{1}, z_{2}, z_{3}\right) \lim _{i \rightarrow+\infty} A^{i}\right) \quad(\text { continuity) } \\
& =F_{3}\left(3 \odot \frac{1}{D}\left[\theta^{2} z_{1}+\theta(1-\theta) z_{2}+(1-\theta)^{2} z_{3}\right]\right) \quad(\text { Lemma } 2.2) \\
& =\frac{1}{D}\left[\theta^{2} z_{1}+\theta(1-\theta) z_{2}+(1-\theta)^{2} z_{3}\right] \quad \text { (reflexivity) }
\end{aligned}
$$

which proves (10).

Now we show that $\theta$ must be $1 / 2$. Strong decomposability implies

$$
F_{3}\left(z_{1}, z_{2}, z_{3}\right)=F_{3}\left(F_{2}\left(z_{1}, z_{3}\right), z_{2}, F_{2}\left(z_{1}, z_{3}\right)\right) \text {. }
$$

By (9) and (10), this identity becomes

$$
\theta(1-\theta)(1-2 \theta)\left(z_{3}-z_{1}\right)=0
$$

that is $\theta=1 / 2$.

Consequently, $M_{2}$ is symmetric. By Lemma 2.3, $M_{n}$ is symmetric for all $n \in \mathbb{N}_{0}$. We then conclude by Theorem 2.2 .

\section{References}

[1] J. Aczél, On mean values, Bull. Amer. Math. Soc. 54 (1948) 392-400.

[2] J. Aczél, Lectures on functional equations and applications, (Academic Press, New York, 1966).

[3] J. Aczél and J. Dhombres, Functional equations in several variables with applications to mathematics, information theory and to the natural and social sciences, (Cambridge Univ. Press, Cambridge, 1989).

[4] G. Bemporad, Sul principio della media aritmetica, Atti Accad. Naz. Lincei (6) 3 (1926) $87-91$.

[5] A.L. Cauchy, Cours d'analyse de l'Ecole Royale Polytechnique, Vol. I. Analyse algébrique, (Debure, Paris, 1821). 
[6] O. Chisini, Sul concetto di media, Periodico di matematiche (4) 9 (1929) 106-116.

[7] B. de Finetti, Sul concetto di media, Giorn. Ist. Ital. Attuari (3) 2 (1931) 369-396.

[8] J.C. Fodor and J.-L. Marichal, On nonstrict means, Aequationes Math. 54 (1997) 308-327.

[9] A.N. Kolmogoroff, Sur la notion de la moyenne, Atti Accad. Naz. Lincei Mem. Cl. Sci. Fis. Mat. Natur. Sez. (6) 12 (1930) 388-391.

[10] J.-L. Marichal, P. Mathonet and E. Tousset, Characterization of some aggregation functions stable for positive linear transformations, Fuzzy Sets and Systems, in press.

[11] J.-L. Marichal and M. Roubens, Characterization of some stable aggregation functions, Proc. Intern. Conf. on Industrial Engineering and Production Management, Mons, Belgium (1993) 187-196.

[12] M. Nagumo, Über eine klasse der mittelwerte, Japan. J. Math., 7 (1930) 71-79.

[13] U. Ricci, Confronti tra medie, Giorn. Economisti e Rivista di Statistica 26 (1915) $38-66$. 